\title{
Citizen contribution to lake water quality observation: SIMILE project Mobile and Web applications
}

\author{
Carrion D. ${ }^{\text {a, }}$, Biraghi C.A. ${ }^{\text {a }}$, Vavassori A. ${ }^{a}$, Pessina E. ${ }^{a}$, Zamboni G. ${ }^{a}$, Bratic G $^{\text {a }}$, and Brovelli M. A. ${ }^{\text {ab }}$ \\ ${ }^{a}$ Dept. of Civil and Environmental Engineering, Politecnico di Milano - Lecco Campus, Via Gaetano previati 1/c, \\ 23900 Lecco, Italy - (daniela.carrion, carloandrea.biraghi, alberto.vavassori, maria.brovelli)@ polimi.it \\ ${ }^{\mathrm{b}}$ Istituto per il Rilevamento Elettromagnetico dell'Ambiente, CNR-IREA, via Bassini 15, 20133 \\ maria.brovelli@polimi.it \\ * Corresponding author
}

Keywords: Citizen Science, Water quality monitoring, Mobile app, Web app

\section{Abstract:}

Lakes represent a crucial resource to be monitored to evaluate and try to mitigate the effects of climate change as well as to preserve the local ecosystem and the human wellbeing. This work is developed in the framework of SIMILE (Informative System for the Integrated Monitoring of Insubric Lakes and their Ecosystems) project, funded by the ItalySwitzerland Interreg programme of the European Commission. SIMILE project is devoted to the monitoring of the lakes of the Insubric area, in particular to lake Maggiore, Como lake and Lugano lake, integrating different surveying and monitoring techniques, namely high frequency in situ measures, remote sensing, citizen science as well as modelling. A significant aim of the project is to overcome the border separation among the two States, facing the same objectives in a synergic way.

In this work, we focus on the Citizen Science component of the lake observation for SIMILE project. The involvement of citizens in the observation and preservation of the environment is very important, first of all because it allows to take advantage of the capillarity of the distribution of people on the territory, which cannot be reached with the traditional in situ measures performed, for example, with the use of buoys. Moreover, it helps attracting the interest of people who become active actors for the protection of their landscape and ecosystem. In fact, the lakes under study are quite large and play a crucial role from the environmental and economic point of view in northern Italy and southern Switzerland.

In the framework of SIMILE project, a Mobile application (Figure 1) has been developed to allow citizens to collect data useful for lake monitoring using their smartphone as well as, when available, additional instruments, such as a thermometer or a Secchi disk. In addition, in order to allow the stakeholders to manage a database of measures, a Web application has been developed. All used and developed tools are free and open source.

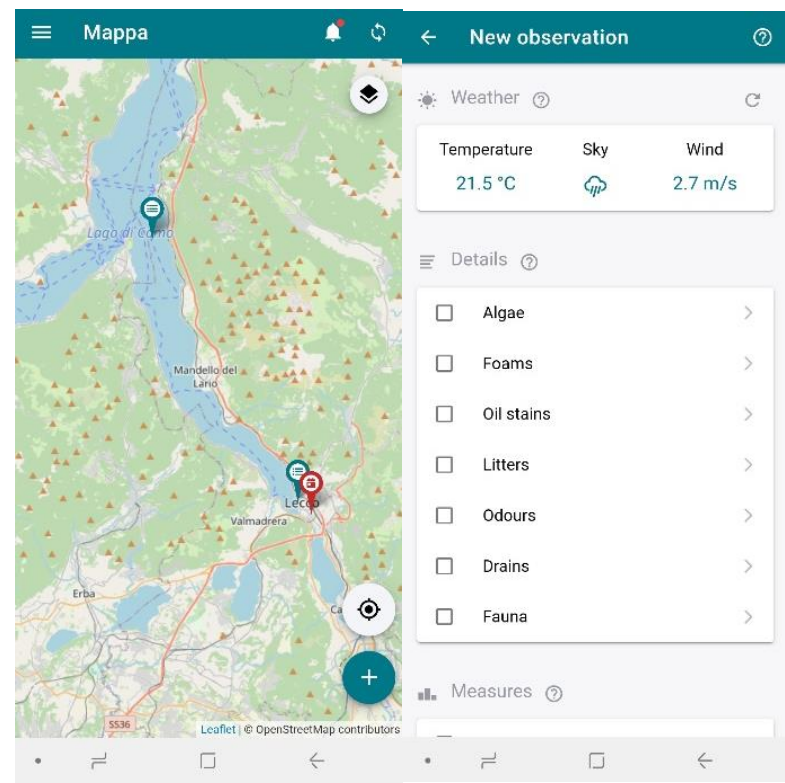

Figure 1. Two screenshots from the Mobile application: on the left, the map showing the observations and allowing to insert a new one, on the right, the interface for submitting a new observation. 
Before developing the application, already existing applications (HydroColor, SecchiApp, Lake Observer) have been explored, in order to check if they could have been exploited in the framework of SIMILE project. None of the selected tools was open source and none was fulfilling completely the goals of the project. For this reason, an ad hoc application has been developed and released both for Android and iOS. The application name is SIMILE, it is user friendly, including in line help and description of every parameter that can be acquired.

The mobile application allows the user to see his as well as other users' observations on a map. With a button on the main screen, he can add a new observation, which can be constituted by a picture only, or include additional details, such as the presence of algae, foams, oil stains, litters, odours, drains, fauna (see Figure 1). It is possible also to add measures, namely Transparency, Temperature, $\mathrm{pH}$, Oxygen, Bacteria. In addition, an open field is made available in order to allow to provide additional information, if needed. The Mobile application allows the stakeholders to advertise events relevant with respect to the lake protection and promotion, such as public cleaning of the lake shores. The application allows also to contact the people in charge (who have agreed in putting their contacts within the app) in case that the observations are worth to be immediately reported to the authorities.

A Web application (see Figure 2) has been designed and developed to efficiently manage the collected data. The Web application is dedicated to authorised stakeholders. It allows to query the data, both based on location and attributes, it allows as well to edit the database of observations, in order to be able to filter the observations as well as to include additional ones directly acquired by researchers and agencies' operators. Also the Web application has been designed to be user friendly. The expert check of the data contributed by citizens is essential, in order to filter not relevant observations. Data can be deleted, but not permanently, in order to be able to retrieve them in case of need. Data of Mobile and Web applications are synchronized, the database can be modified on either side without problems of misalignment.

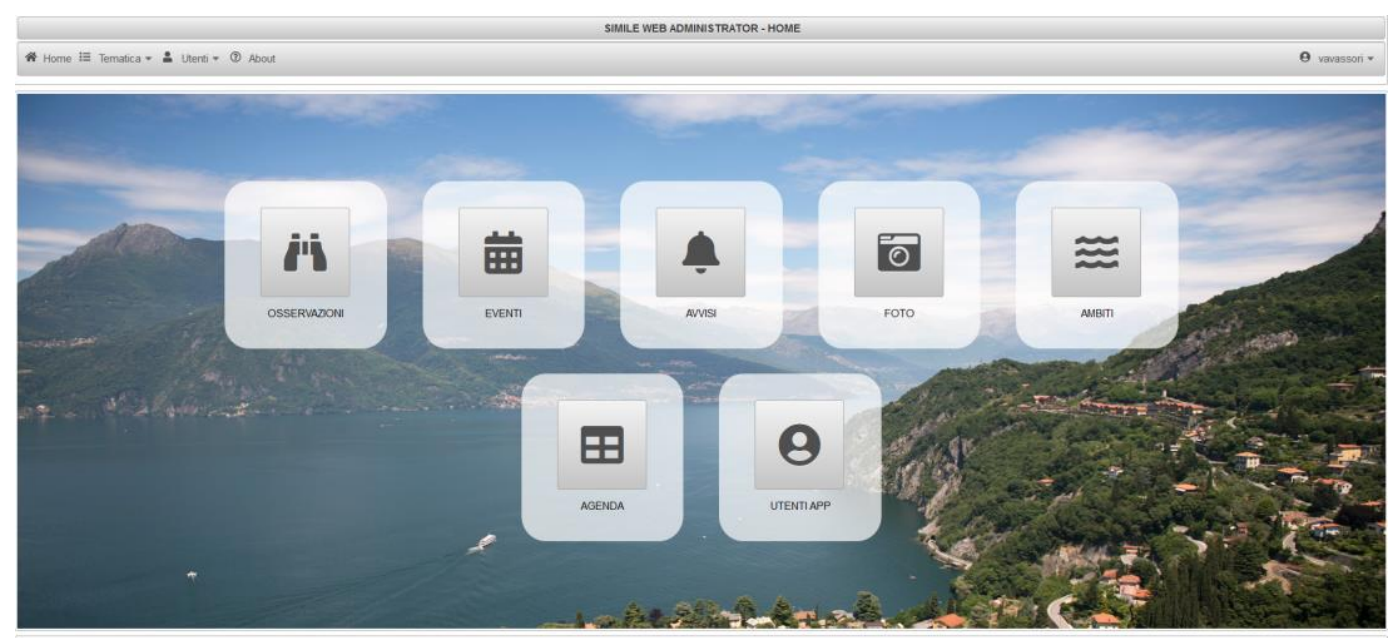

Figure 2. Home page of the Web application.

Both applications have been developed in cooperation with the project partners, including research centre and academic institutions (CNR-IRSA and SUPSI), local agencies (ARPA Lombardia) and local administrations (Regione Lombardia and Ticino Canton) working on the lakes management and preservation.

The applications have been presented to the project partners and to potential users through several initiatives. First of all, a course has been devoted to the project partners as well as to other interested stakeholders in order to teach how to use both applications. In addition, the citizen science activities of SIMILE project include the involvement of schools and associations acting in interaction with the lake (fishermen, diving associations, associations dedicated to the preservation of the environment, ...) promoting the mobile app with initiatives to teach how to contribute to lake water quality monitoring. The promoting activities have been slowed down due to the pandemic, however online meetings and seminars have been organised with school teachers and students. During 2021 Spring several secondary schools classes started the collection of data through the Mobile app.

In the next phase of the project the promotion and cooperation with teaching activities with the schools will continue and the analysis of the collected data will proceed.

\section{ACKNOWLEDGEMENTS}

This work is part of project SIMILE (Informative System for the Integrated Monitoring of Insubric Lakes and their Ecosystems) and as such it is financed by the European Commission in the frame of Interreg Italy-Switzerland 20142021 programme. 